\title{
The Analytical Description of Projectile Motion of Cricket Ball in a Linear Resisting Medium the Storm Force
}

\author{
Siranee Kuaykaew ${ }^{1 \mathrm{a}}$, Supoj Kerdmee ${ }^{1 \mathrm{~b}}$, Paitoon Banyenugam ${ }^{1 \mathrm{c}}$, \\ Piyarut Moonsri ${ }^{2 \mathrm{~d}}$, and Artit Hutem ${ }^{1 \mathrm{e}^{*}}$ \\ ${ }^{1}$ Physics Division, Faculty of Science and Technology, Phetchabun Rajabhat University, \\ Phetchabun, Thailand 67000 \\ ${ }^{2}$ Chemistry Division, Faculty of Science and Technology, Phetchabun Rajabhat University, \\ Phetchabun, Thailand 67000 \\ aP7925@outlook.com, 'bupojkerdmee@homail.com,,Paitoonbanyenugam@outlook.com, \\ dpiyarutto@hotmail.com, ${ }^{\mathrm{e}}$ magoohootem@yahoo.com \\ * Corresponding author. E-mail: magoohootem@yahoo.com, \\ Fax: 056-717-123, Mobile: 086-9909536
}

Keywords: Velocity, Projectile Motion, Storm Force, Damped Coefficient

\begin{abstract}
A detailed work based on the second-order ordinary differential equation is presented to solve oscillation in the trajectory projectile motion of cricket ball for damped alternating external force $\left(f_{0}\right)$ problems. This paper purpose to compute the distance time depends horizontal and the distance time depends vertical. The parabolic path of trajectories for a projectile motion of cricket ball increase oscillation with the value of parameter $\lambda$ and $f_{0}$ is the storm force.
\end{abstract}

\section{Introduction}

Peter Coutis used the quadratic drag force to calculate the distance vertical $(y(t))$ motion and distance horizontal $(\mathrm{x}(\mathrm{t}))$ in projectile motion of a cricket ball. Sean M. Stewart consider the time of flight, range and the angle which maximizes the range of a projectile motion in a linear resisting medium are expressed in analytic form in terms of the recently defined Lambert $\mathrm{W}$ function is to be the inverse of $\boldsymbol{W} e^{W}=\boldsymbol{z}$. Jeffrey Leela et. al, explores the various equation associated with the movement of the projectile motion for ball in flight and they use the velocity depends on $C_{D}$ and also the drag force couples the equation for the horizontal and vertical component of the velocity see equation (1) and equation (2), where $\boldsymbol{C}_{\boldsymbol{D}}$ is the drag coefficient

\section{Projectile motion of cricket ball below external force $\left(f_{0} e^{-\lambda t} \cos (\omega t)\right)$}

The parameters governing the projectile of motion of a cricket ball are the launch angle $(\boldsymbol{\theta})$, the speed at which the ball leaves the bat $\left(\boldsymbol{v}_{\mathbf{0}}\right)$, and the linear drag coefficient per unit mass $(\boldsymbol{\beta})$. If we assume that the only forces acting on the cricket ball in flight are gravity drag force and external forces $\left(f_{0} e^{-\lambda t} \cos (\omega t)\right)$, Newton's second law implies

$$
m \ddot{x}=-C_{D} \dot{x} \cos (\theta)
$$

and

$$
m \ddot{y}=-C_{D} \dot{y} \sin (\theta)-m g-f_{0} e^{-\lambda t} \cos (\omega t)
$$

The cricket ball's motion is best described by separating it into horizontal $(\mathrm{x}(\mathrm{m}))$ and vertical $(\mathrm{y}(\mathrm{m}))$ components as we have already emphasized, the horizontal motion dependent of the vertical motion and then applying the kinematic equation. Here $\dot{x}(t)$ is the velocity time dependent and $\ddot{x}(t)$ is 
the acceleration time dependent, $\boldsymbol{C}_{D}$ is the drag coefficient, $\boldsymbol{\lambda}$ is the damped coefficient. Multiplying the above equation by $\mathbf{1} / \boldsymbol{m}$ gives

$$
\ddot{x}(t)+\beta \dot{x}(t) \cos (\theta)=0
$$

and

$$
\ddot{y}(t)+\beta \dot{y} \sin (\theta)=-\left(g+F_{0} e^{-\lambda t} \cos (\omega t)\right),
$$

where $\beta=C_{D} / \boldsymbol{m}, \boldsymbol{F}_{\mathbf{0}}=\boldsymbol{f}_{\mathbf{0}} / \boldsymbol{m}$. We use the auxiliary equation find solution of equation (3)

$$
x(t)=A+B e^{-\beta t \cos (\theta)} .
$$

Here, $\boldsymbol{A}$ and $\boldsymbol{B}$ are constants which can be determined from the initial condition $\boldsymbol{x}(\mathbf{0})=\mathbf{0}, \dot{x}(\mathbf{0})=\boldsymbol{v}_{\mathbf{0}} \cos (\boldsymbol{\theta})$. A solution to equation (3) is

$$
x(t)=\frac{v_{0}}{\beta}\left(1-e^{-\beta t \cos (\theta)}\right)
$$

From the above result, we gives a time-dependent expression for $\boldsymbol{x}$, which is the horizontal distance travelled by the cricket ball in time $t$. Rearrangement of equation (6) provides

$$
t=\ln \left[\frac{v_{0}-\beta x(\mathrm{t})}{v_{0}}\right]^{\frac{-1}{\beta \cos (\theta)}}
$$

Find the general solution of the inhomogeneous of equation $(4), y(t)=y_{C}(t)+y_{P}(t)$. Find the fundamental solution to the homogeneous equation the characteristic equation is $y_{C}(t)=C+D e^{-\beta t \sin (\theta)}$. Here, $y_{1}(t)=1, y_{2}(t)=e^{-\beta t \sin (\theta)}$. Compute their Wronskian,

$$
\begin{array}{ll}
W=-\beta \sin (\theta) e^{-\beta t \sin (\theta)}, & W_{1}=\left(g+F_{0} e^{-\lambda t} \cos (\omega t)\right) e^{-\beta t \sin (\theta)}, \\
W_{2}=-\left(g+F_{0} e^{-\lambda t} \cos (\omega t)\right) . &
\end{array}
$$

We compute the function $\boldsymbol{u}_{1}(t), \boldsymbol{u}_{2}(t)$, we obtain the particular solution is

$$
\begin{aligned}
y_{P}(t)= & \frac{F_{0} e^{-\lambda t}(\lambda \cos (\omega \mathrm{t})-\omega \sin (\omega \mathrm{t}))}{\beta \sin (\theta)\left(\lambda^{2}+\omega^{2}\right)}-\frac{g t}{\beta \sin (\theta)}+\frac{g}{\beta^{2} \sin ^{2}(\theta)} \\
& +\frac{\boldsymbol{F}_{0} e^{-\lambda t}((\beta \sin (\theta)-\lambda) \cos (\omega \mathrm{t})+\omega \sin (\omega \mathrm{t}))}{\beta \sin (\theta)\left((\beta \sin (\theta)-\lambda)^{2}+\omega^{2}\right)}
\end{aligned}
$$

The general solution is

$$
\begin{aligned}
y(t)= & C+D e^{-\beta t \sin (\theta)}-\frac{g t}{\beta \sin (\theta)}+\frac{F_{0} e^{-\lambda t}(\lambda \cos (\omega t)-\omega \sin (\omega t))}{\beta \sin (\theta)\left(\lambda^{2}+\omega^{2}\right)} \\
& +\frac{F_{0} e^{-\lambda t}((\beta \sin (\theta)-\lambda) \cos (\omega t)+\omega \sin (\omega t))}{\beta \sin (\theta)\left((\beta \sin (\theta)-\lambda)^{2}+\omega^{2}\right)}
\end{aligned}
$$

With the initial condition for $y(t)$, i.e. $y(0)=0, \dot{y}(0)=v_{0} \sin (\theta)$ the expression for $y(t)$ becomes 


$$
\begin{aligned}
y(t)= & {\left[\left(1-e^{-\beta t \sin (\theta)}\right)\left(\frac{v_{0}}{\beta}+\frac{g}{\beta^{2} \sin ^{2}(\theta)}\right)\right]-\frac{F_{0} e^{-\beta t \sin (\theta)}(\lambda-\beta \sin (\theta))}{\beta \sin (\theta)\left((\beta \sin (\theta)-\lambda)^{2}+\omega^{2}\right)} } \\
& -\frac{g t}{\beta \sin (\theta)}+\frac{F_{0} e^{-\lambda t}(\lambda \cos (\omega \mathrm{t})-\omega \sin (\omega \mathrm{t}))}{\beta \sin (\theta)\left(\lambda^{2}+\omega^{2}\right)}-\frac{F_{0} \lambda}{\beta \sin (\theta)\left(\lambda^{2}+\omega^{2}\right)} \\
& +\frac{F_{0} e^{-\lambda t}((\beta \sin (\theta)-\lambda) \cos (\omega \mathrm{t})+\omega \sin (\omega \mathrm{t}))}{\beta \sin (\theta)\left((\beta \sin (\theta)-\lambda)^{2}+\omega^{2}\right)}
\end{aligned}
$$

\section{Results and Discussion}

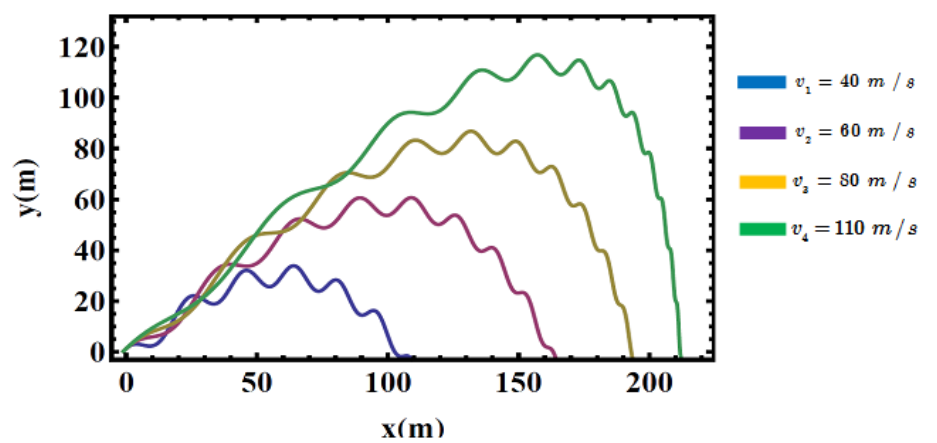

Fig.1 A trajectories projectile of motion for a cricket ball struck at an angle of calculation of $45^{\circ}$ in the presence of a linearized drag force by vary the initial velocity.

From figure 1 we illustrate magnitude of the $\mathrm{x}(\mathrm{t})$ horizontal distance time dependent and $\mathrm{y}(\mathrm{t})$ vertical distance time dependent increase from $100 \mathrm{~m}$ to $210 \mathrm{~m}$ with increasing the velocity of trajectories projectile of motion for a cricket ball.

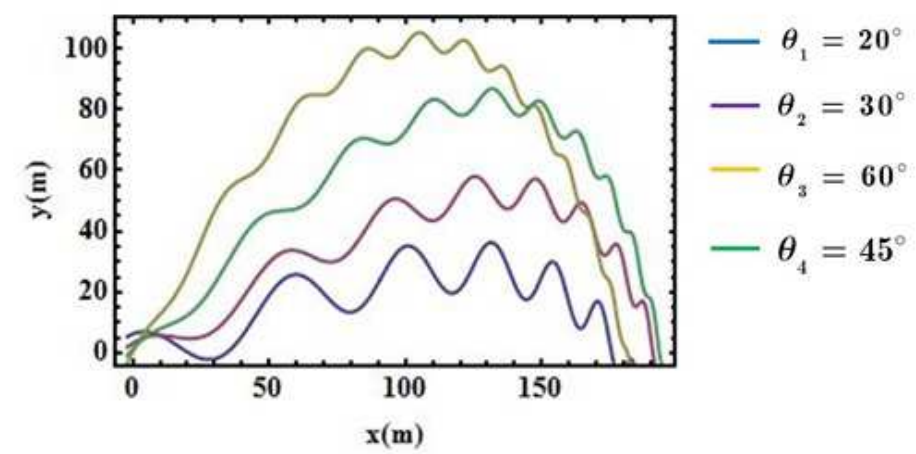

Fig.2 A trajectories projectile of motion for a cricket ball struck at an angle of calculation of $45^{\circ}$ in the presence of a linearized drag force by vary an angle.

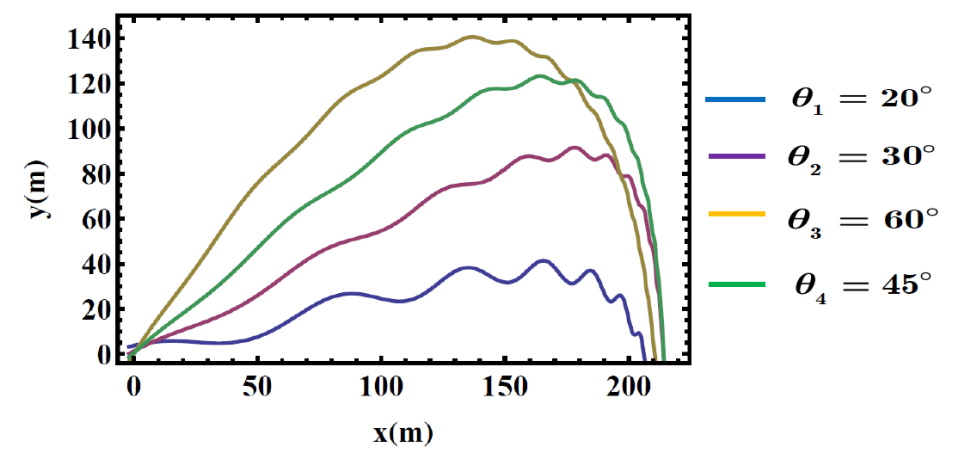

Fig.3 A schematic diagram for behavior of a trajectories projectile of motion for a cricket ball in case of $\boldsymbol{\lambda}=\mathbf{0 . 0 5}$ is the damped coefficient. 


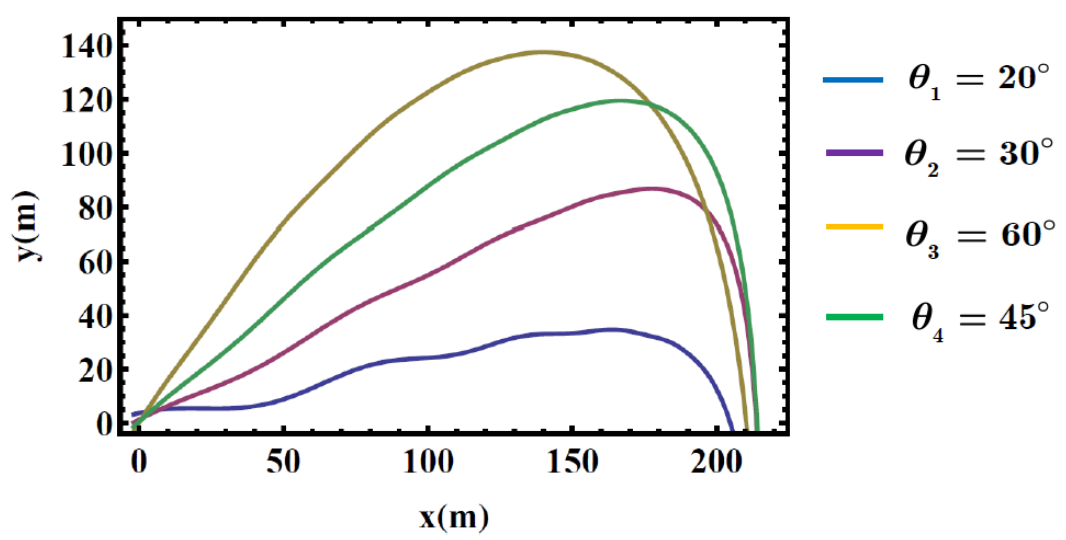

Fig.4 A schematic diagram for behavior of a trajectories projectile of motion for a cricket ball in case of $\boldsymbol{\lambda}=\mathbf{0 . 9 9}$ is the damped coefficient.

From figure 3 and 4 , If the value of parameter $\boldsymbol{\lambda}$ is small the parabolic path of trajectories for projectile motion of a cricket ball will increase small oscillation and the value of parameter $\boldsymbol{\lambda}$ is large the parabolic path of trajectories for projectile motion of a cricket ball will decrease small oscillation as figure 4.

\section{Conclusions}

The parabolic path of trajectories for a projectile motion of cricket ball increase oscillation with the value of parameter $\lambda$ and $f_{0}$ is the storm force. When at angle $60^{\circ}$ the high parabolic path of cricket ball more than the angle $30^{\circ}$ it is influence due to the large storm force affection $\mathrm{x}(\mathrm{t})$ horizontal distance time dependent and $\mathrm{y}(\mathrm{t})$ vertical distance time dependent little as figure 2 .

\section{References}

[1] R.A. Serway et. al., Physics for Scientists and Engineers with Modern Physics, Fifth Edition, saunders college publishing, 2000, pp.82-90.

[2] K.F. Riley et. al., Mathematical Method for Physics and Engineering, Third Edition, Cambridge, 2006, pp.490-529.

[3] S. Thornton and J.B. Marion, Classical Dynamics of Particles and System, Fifth Edition, Thomson publishing, 2002, pp.99-135.

[4] Peter Coutis, Modeling the projectile motion of a cricket ball, INT. J. MATH. EDUC. SCI. TECHNOL., Vol. 29 No. 6, pp 789-798.(1999)

[5] Sean M. Stewart, An analytic approach to projectile motion in a linear resisting medium, INT. J. MATH. EDUC. SCI. TECHNOL., Vol. 37 No. 4, 266 pp 411-431.(2006)

[6] Jeffrey Leela et. al, Modelling the flight characteristics of a soccer ball, Lat. Am. J Phys. Educ. Vol. 8 No.4 pp. 4505-1 - pp.4505-5., (2016) 